\title{
Controlling of Optical Band Gap of Allyl Diglycol Carbonate Polymer with Ultraviolet Laser Radiation
}

\author{
W.A. Farooq ${ }^{a, *}$, M.R. BAig $^{a}$, A. Fatehmulla ${ }^{a}$, M.S. Al-SAlhi ${ }^{a}$, S.S. Al-Ghamdi ${ }^{a}$ \\ AND F. YAKUPHANOGLU ${ }^{b}$ \\ ${ }^{a}$ Department of Physics and Astronomy, College of Science, P.O. Box 2455, King Saud University \\ Riyadh 11451, Kingdom of Saudi Arabia \\ ${ }^{b}$ Department of Physics Faculty of Science, Firat University, Elazı $\breve{g}$, Turkey
}

(Received January 30, 2012; in final form May 17, 2012)

\begin{abstract}
The effects of ultraviolet laser radiation on the structure and optical properties of allyl diglycol carbonate have been investigated. The allyl diglycol carbonate samples were irradiated with $266 \mathrm{~nm}$ with different power densities from Nd:YAG laser. The bulk etch rate enhancement and enlargement of track diameter clearly indicate that allyl diglycol carbonate is significantly affected by UV laser. The laser-irradiated allyl diglycol carbonate samples showed a decrease in the optical band gap with increasing laser power density. The obtained results indicate that the optical band gap of the alpha irradiated polymer is varied from $4.10 \mathrm{eV}$ to $2.65 \mathrm{eV}$ by UV laser irradiation.
\end{abstract}

DOI: 10.12693/APhysPolA.123.106

PACS: 78.20.-e, 61.82.Pv, 61.80.Ba

\section{Introduction}

Allyl diglycol carbonate (ADC) or CR-39 is a plastic polymer. It is transparent in visible spectrum. It was the 39th formula of a thermosetting resin developed by the Columbia Resin project in 1940. It combines an exceptional range of qualities which are not available in other plastic transparent materials [1]. Stanton was among the co-developers of CR-39 monomer in 1940. The patent was registered in October 1940. CR-39 monomer was first used commercially to help create glass-reinforced plastic fuel tank for B-17 bomber aircraft in World War II, reducing weight and increasing range of bomber. It can withstand for the small hot sparks from welding where glass cannot do. It is also resistant to most of solvents and to material fatigue [2]. Due to its optical properties it is being used for lenses in optical glasses.

In recent decades, CR-39 has been widely used as nuclear track detector for detection of charged particles. It has also been used to monitor concentration of radon gas by recording alpha particles emitted from radon and its progeny. When CR-39 is exposed to charged particles, it produces a latent damage trail due to low linear energy transfer (LLET), called "latent track" which can be made visible under an optical microscope when etched in a suitable reagent under optimum conditions. The irradiation in track detectors yields cross-linking and chain scission. Due to cross-linking, etch rate decreases. If the probability of cross-linking reaction is larger than chain scission, the material would become hardened. In chain scission process, emission of atoms and molecules $(\mathrm{CO}$, $\mathrm{CO}_{2}$, and $\mathrm{H}_{2}$ ) occurs as a result of cut in the long chain. This process softens the material which increases etch rate.

*corresponding author; e-mail: wafarooq@hotmail.com
It has been reported in literature that track registration properties of latent tracks are affected by exposing such detectors to different type of radiations [3-7]. The track developments in solid state nuclear track detectors (SSNTDs) depend strongly on the ratio of track etch rate to the bulk etch rate $\left(V=V_{\mathrm{T}} / V_{\mathrm{B}}\right)$. The quantities $V_{\mathrm{T}}$ and $V_{\mathrm{B}}$ are dependent on etching parameters such as etching time, temperature and concentration of solution. Durrani and Bull [8] have reported that track diameter is directly proportional to the bulk etch velocity $V_{\mathrm{B}}$.

$$
D=2 V_{\mathrm{B}} t \sqrt{\frac{V_{\mathrm{T}}-V_{\mathrm{B}}}{V_{\mathrm{T}}+V_{\mathrm{B}}}}, \quad D \simeq 2 V_{\mathrm{B}} t
$$

Many investigations have been carried out for UV exposures to alpha irradiated CR-39 in the last few years. Jaleh et al. in 2004 [9] exposed CR-39 to $\mathrm{KrF}$ laser $(248 \mathrm{~nm})$, then irradiated the samples with alpha particles. They found that mean track diameters decrease due to cross-linking and it occurs at wavelength lower than $315 \mathrm{~nm}$. The registration efficiency decreases at higher UV laser doses.

Parvin et al. in 2005 [10] investigated the optical penetration depth for CR-39 at $308 \mathrm{~nm}$ from XeCl laser, at $248 \mathrm{~nm}$ and $193 \mathrm{~nm}$ from $\mathrm{XeCl}$ and $\mathrm{ArF}$ lasers. They concluded that optical penetration depth of CR-39 decreases with increasing energy of the photons. They also showed that diameter of tracks decreases as the exposure doses increase, thus indicating UV laser exposure leads to skin cross-linking and hardening of the surface polymer structure. Dwaikat et al. in 2007 [11], exposed CR-39 to pulsed laser of wavelength of $266 \mathrm{~nm}$ from indium-doped yttrium aluminum garnet (UV-In:YAG) laser system to investigate the effects on etching characteristics of Japanese CR-39 at various energy intensities.

Up to the best of our knowledge, no research has been carried out on the variation of optical band gaps for UV exposed alpha irradiated CR-39 samples at different en- 
ergies and different time exposures. Such study may lead to investigate the semiconductor behavior of alpha irradiated CR-39 after UV exposure. In the present work, we have taken absorption spectra of alpha+laser irradiated samples. Then optical bandgaps of the samples were calculated.

\section{Experimental details}

Samples of dimension $2 \mathrm{~cm} \times 2 \mathrm{~cm}$ were cut from CR-39 sheet of thickness $500 \mu \mathrm{m}$, density $1.32 \mathrm{~g} / \mathrm{cm}^{3}$ manufactured by Pershore Ltd. England. The samples were irradiated at normal incidence with alpha particles using americium-241 source. The sample-source distance was $2 \mathrm{~cm}$ and the irradiation period was $5 \mathrm{~s}$ for each sample. The alpha-irradiated samples are divided into two sets $\mathrm{A}$ and $\mathrm{B}$ in addition to one sample used as a reference. The two sets of samples were exposed to laser beam from Nd:YAG Brillant-Quantel $Q$-switched laser system with pulse width $8 \mathrm{~ns}$ (FWHM). Set A was exposed at wavelength $266 \mathrm{~nm}$ with $10 \mathrm{~Hz}$ repetition rate. The energy of the pulsed laser was fixed at $80 \mathrm{~mJ}$ and the time of exposure was varied. Set B was exposed at the same wavelength keeping $10 \mathrm{~Hz}$ repetition rate for $5,10,15$, 35 , and $45 \mathrm{~mJ}$ energies for $180 \mathrm{~s}$.

After alpha (reference sample) and (alpha+laser) irradiation (sets $\mathrm{A}$ and $\mathrm{B}$ ), the samples were chemically etched in $6 \mathrm{~N}$ of $\mathrm{NaOH}$ at a temperature of $70^{\circ} \mathrm{C}$ for a period of three hours. The track diameter was measured for all samples using a 400X optical microscopy. Optical absorption studies were carried out using UV-Vis spectroscopy (Perkin Elmer spectrometer - Lambda 40) in the wavelength of $250 \mathrm{~nm}$ to $600 \mathrm{~nm}$ with $1 \mathrm{~nm}$ accuracy.

\section{Results and discussion}

Images of alpha irradiated (reference sample) and observed changes in alpha + laser irradiated, set A and set B samples, taken under microscope are shown in Fig. 1, 2, and 3 respectively. As the samples are irradiated with

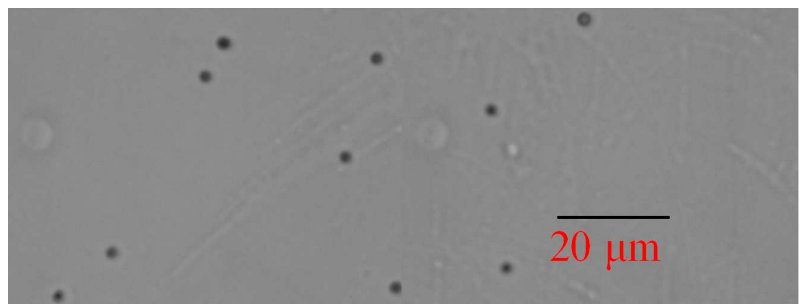

Fig. 1. Surface morphology of alpha irradiated sample of CR-39.

UV laser, the track diameter increases with increasing laser energy and exposure time. At higher power densities, the track diameters are merged in each other which show change in structure of the whole exposed sample area. The graph of variation in track diameter with increasing laser power density, shown in Fig. 4, also indicates a linear increase of track diameters. Figure 5

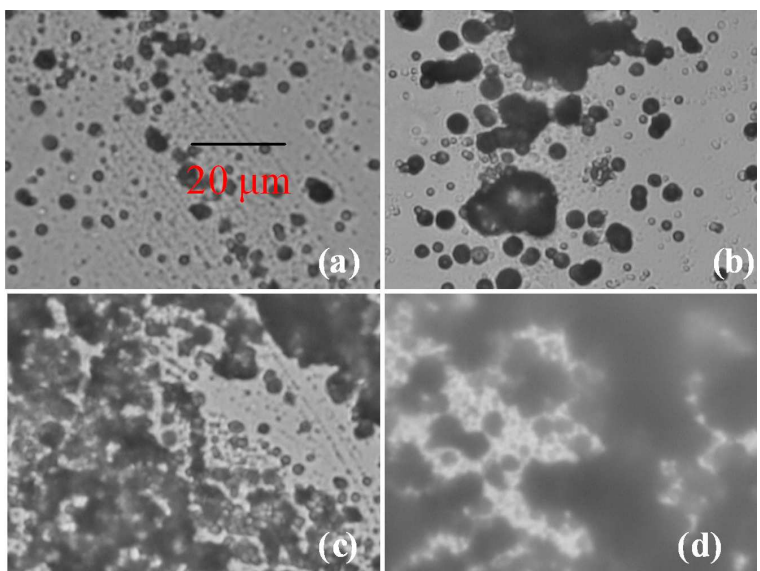

Fig. 2. Surface morphology of alpha $+U V$ irradiated samples at $35 \mathrm{~mJ}$ for (a) $60 \mathrm{~s}$, (b) $120 \mathrm{~s}$, (c) $180 \mathrm{~s}$, (d) $300 \mathrm{~s}$.

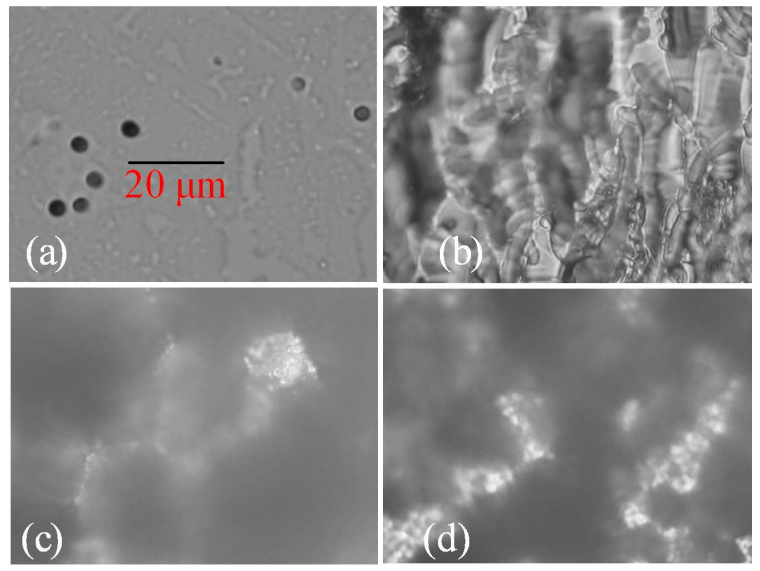

Fig. 3. Surface morphology of alpha $+\mathrm{UV}$ irradiated samples at (a) $10 \mathrm{~mJ}$, (b) $15 \mathrm{~mJ}$, (c) $35 \mathrm{~mJ}$, (d) $45 \mathrm{~mJ}$ for $180 \mathrm{~s}$.

presents the bulk etch rate $\left(V_{\mathrm{B}}\right)$ as a function of laser power density. The bulk etch rate increases linearly with increasing laser exposure time. Softening behavior of CR-39 after alpha+laser irradiation is also obvious from increasing trend in bulk etch rate with increasing laser power density which is shown in Fig. 5 .

At the higher value of energy, $45 \mathrm{~mJ}$, the bulk etch rate at the exposed area reaches to $0.00344 \mu \mathrm{m} / \mathrm{s}$ and at this

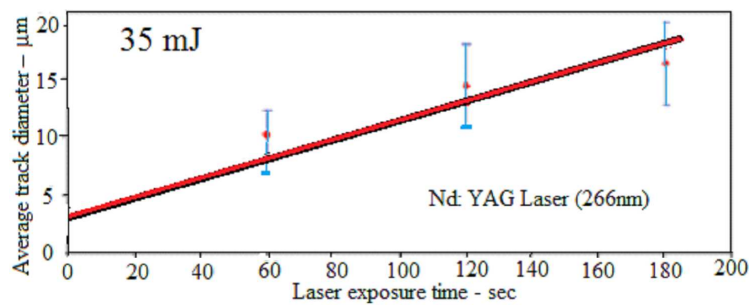

Fig. 4. Variation in track diameter with laser exposure time. 


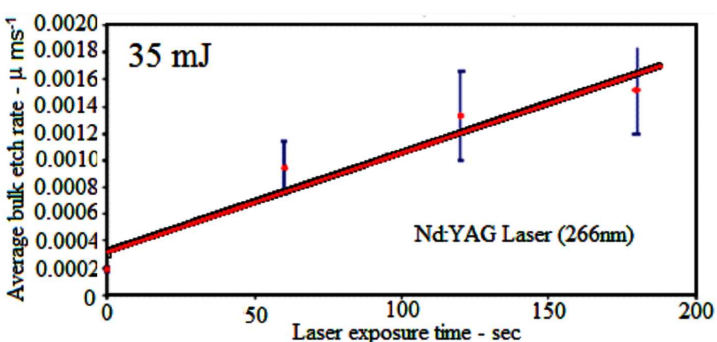

Fig. 5. Bulk etch rate $\left(V_{\mathrm{B}}\right)$ vs. laser exposure time at wavelength $266 \mathrm{~nm}$.

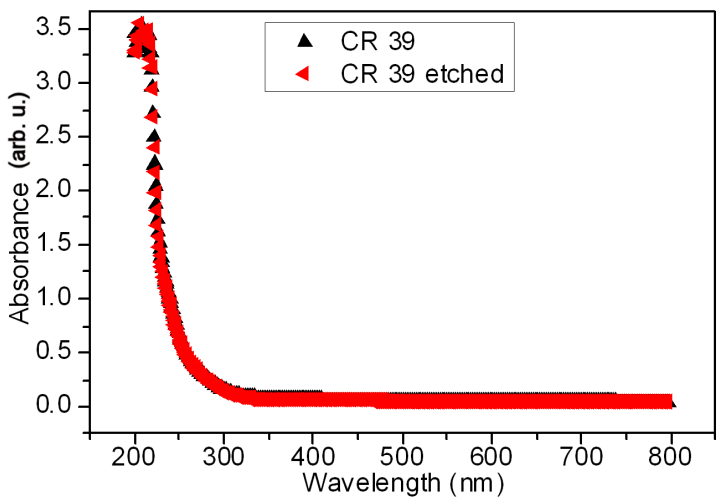

Fig. 6. Absorbance curves of CR-39 and etched samples (without irradiation).

value of energy and the exposure time the average bulk etch rate is reached about 9 times of the average bulk etch rate as compared to the non-exposed area. The effect of UV laser radiation on the latent tracks in CR-39 is both wavelength and exposure time dependent. The bulk etch rate enhancement and enlargement of track diameter clearly indicates that this sample is significantly affected by UV laser irradiation. All these behaviors indicate that the material has become softened with increasing exposure time and energy density.

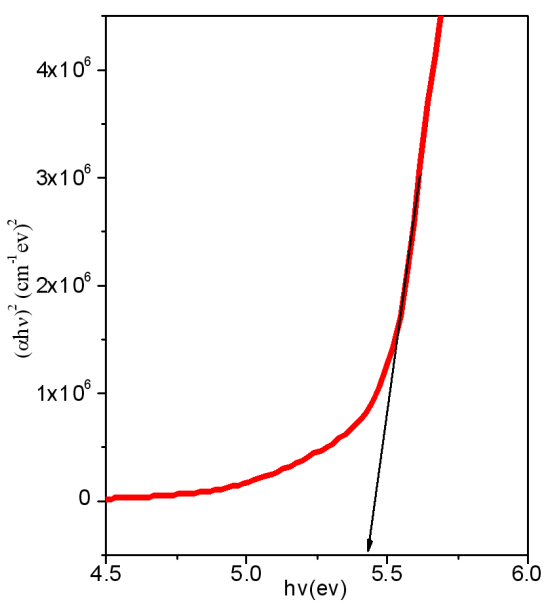

Fig. 7. Plot of $(\alpha h \nu)^{2}$ vs. $h \nu$ for CR-39 sample.
We have taken the absorption spectra of CR-39 (without alpha+laser irradiation) and etched CR-39 which are shown in Fig. 6. As seen in Fig. 6, the fundamental absorption edge for CR-39 as well as etched samples is around $229 \mathrm{~nm}$ and there are no other absorption bands in the studied wavelength range. Figure 6 also shows that there is an exponential rise in the absorption towards the edge and there is no significant change in the absorption edge of reference and etched samples. Figure 7 shows the plot of $(\alpha h \nu)^{2}$ versus $h \nu$ for CR-39 sample. Extrapolating the straight parts of these relations to the $h \nu$ axis yields a forbidden energy gap $\left(E_{\mathrm{g}}\right)$. All the values were tabulated (Tables I and II) and compared.

TABLE I

Variation of band gap values of CR-39 with different laser exposure energy at $180 \mathrm{~s}$.

\begin{tabular}{c|c|c}
\hline \hline S. no. & $\begin{array}{c}\text { Laser exposure energy } \\
{[\mathrm{mJ}]}\end{array}$ & $\begin{array}{c}\text { Band gap } \\
{[\mathrm{eV}]}\end{array}$ \\
\hline 1 & 5 & 4.10 \\
2 & 15 & 3.95 \\
3 & 35 & 4.05 \\
4 & 45 & 3.80
\end{tabular}

TABLE II

Variation of band gap values of CR-39 with different laser exposure time at $35 \mathrm{~mJ}$ exposure energy.

\begin{tabular}{c|c|c}
\hline \hline S. no. & $\begin{array}{c}\text { Laser exposure time } \\
{[\mathrm{s}]}\end{array}$ & $\begin{array}{c}\text { Band gap } \\
{[\mathrm{eV}]}\end{array}$ \\
\hline 1 & 60 & 3.40 \\
2 & 120 & 3.82 \\
3 & 180 & 3.20 \\
4 & 300 & 2.65
\end{tabular}

The absorption spectra of the samples of set A and B are shown in Fig. 8 and Fig. 9. The relationship between absorption coefficient $\alpha$ and optical band gap $E_{\mathrm{g}}$ of material is expressed by the following relation [12]:

$$
(\alpha h \nu)=C\left(h \nu-E_{\mathrm{g}}\right)^{n},
$$

where $C$ is an energy-independent constant and $n$ is a constant which determines type of optical transitions. The values of the direct optical band gap $E_{\mathrm{g}}$ can be obtained from the intercept of $(\alpha h \nu)^{2}$ vs. $h \nu$ curves shown in Fig. 10 and Fig. 11 for samples of set A and B, respectively $[13,14]$. The obtained values of optical band gaps of the samples are given in Tables I and II for set A and $\mathrm{B}$, respectively. As seen in Fig. 8 and 9 , the absorption edge for all the curves is shifted towards longer wavelength upon (alpha $+\mathrm{UV}$ laser) irradiated samples with $35 \mathrm{~mJ}$ energy for different exposure time as well as upon (alpha $+\mathrm{UV}$ laser) irradiated samples at different energies for $180 \mathrm{~s}$. This is a clear indication that the forbidden band gap is decreased with increase of either exposure time or different energies. Also Figs. 8 and 9 show that there is an exponential rise in the absorption towards the edge. The absorption edges are not sharply 
defined, signifying the amorphous nature of the films. Similar observations of decrease in $E_{\mathrm{g}}$ with increase of the exposure time of UV-irradiation on polycarbonate films was reported by Migahed and Zidan in 2006 [15].

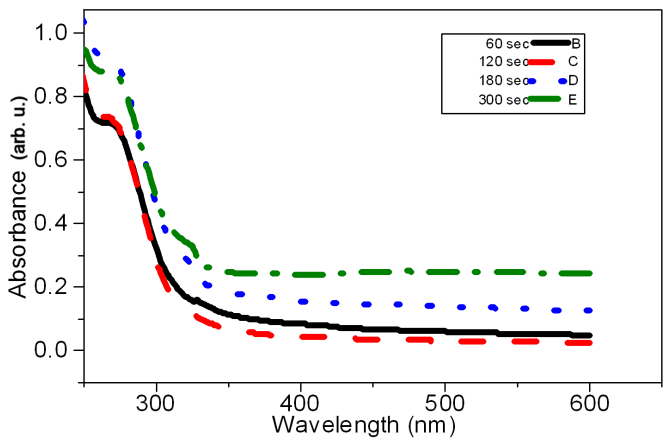

Fig. 8. Absorbance curves of alpha $+\mathrm{UV}$ laser irradiated samples with $35 \mathrm{~mJ}$ energy for different exposure time.

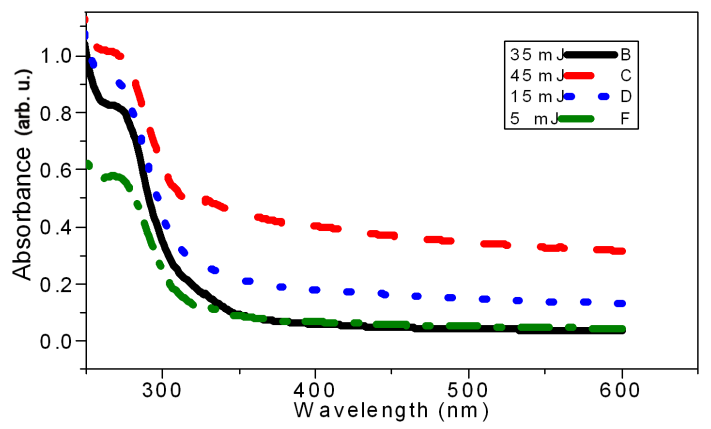

Fig. 9. Absorbance curves of (alpha+UV laser) irradiated samples at different energies for $180 \mathrm{~s}$.

It is clear from Fig. 8 and Fig. 9 that at lower laser power density, there are two absorption peaks at $230 \mathrm{~nm}$ and $269.7 \mathrm{~nm}$ but at high laser power density, a third absorption peak appears at $326.8 \mathrm{~nm}$. Absorption also increases with increasing laser power density. The reason for this is not very much clear at the moment and needs further investigation in this area. We can predict that at high power density observed changes might be due to scission of chains in the polymer.

It is obvious from Fig. 8 and Fig. 9 that the absorption at $266 \mathrm{~nm}$ is high. Due to this absorption, there must be decomposition of molecules which may end up with chain scission in the material. Because of chain scission, material has become softened $[11,12]$ and band gap of the material has shifted towards semiconductor range at high power density of laser. This band-gap shift towards semiconductor range is also obvious from Table I, Table II, Fig. 10 and Fig. 11 related to band-gaps of the samples. Figure 10 shows variation of optical band gap values with different laser energies for $180 \mathrm{~s}$ exposure time, whereas Fig. 11 shows the variation of optical band gap values at $35 \mathrm{~mJ}$ energy of $266 \mathrm{~nm}$ wavelength laser with differ-

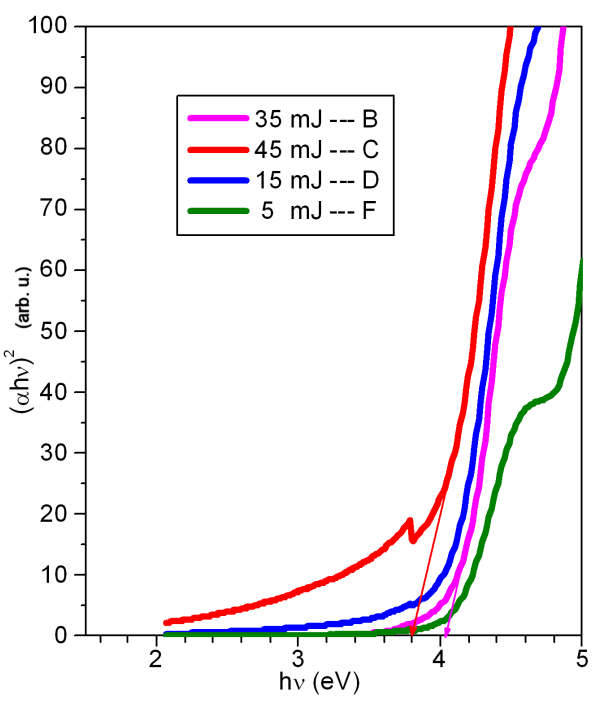

Fig. 10. Plots of $(\alpha h \nu)^{2}$ vs. $h \nu$ for irradiated samples at different energies for $180 \mathrm{~s}$.

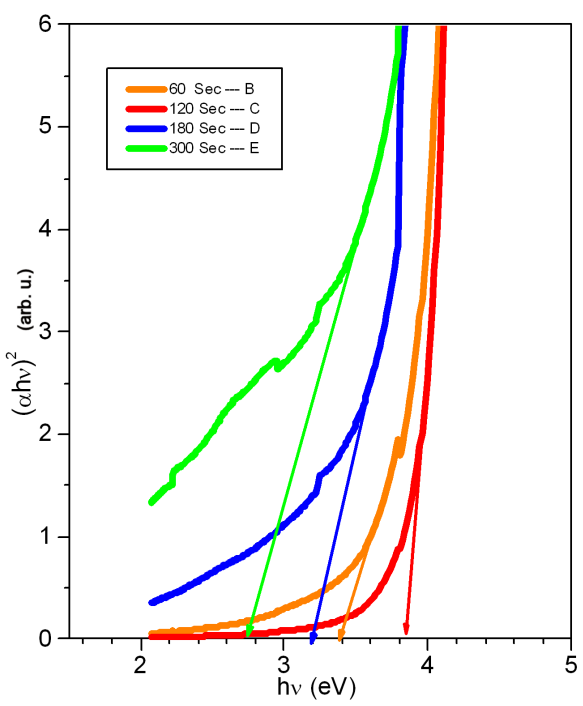

Fig. 11. Plots of $(\alpha h \nu)^{2}$ vs. $h \nu$ for irradiated samples at $35 \mathrm{~mJ}$ energy of $266 \mathrm{~nm}$ laser with different exposure times.

ent exposure times. Due to the small size of the samples, it was not possible to measure the resistance of the irradiated samples. Further investigations are required to study the semiconductor behavior of such irradiated CR-39 samples.

It can be evaluated that the obtained optical band gap values by this method are suitable for many scientific studies and technological applications, such as transparent electrodes, solar cells and piezoelectric devices.

\section{Conclusions}

We have reported the shift in band-gaps towards semiconductor range in alpha irradiated ADC samples after 
exposure to ultraviolet radiation $(266 \mathrm{~nm})$ at high power densities from Nd:YAG laser. An important change in the $E_{\mathrm{g}}$ values among the CR-39 and (alpha+UV) irradiated samples was noticed. This behavior is estimated with band-bap calculation from absorption curves at different laser power densities. It is also concluded that the shift in band-gap towards semiconductor range is due to softening of material which happens with chain scission of polymer. Further investigation is needed in this area to develop complete picture of such changes in irradiated samples of ADC.

\section{Acknowledgments}

This project was supported by King Saud University Deanship of Scientific Research, College of Science Research Center.

\section{References}

[1] R.M. Cassou, E.V. Benton, Nucl. Track Detect. 2, $173(1978)$

[2] Wikipedia: CR-39.

[3] R.A. Akber, K. Nadeem, C.A. Majid, A. Hussain, N. Zaman, M.A. Chaudhary, H.A. Khan, Nucl. Instrum. Methods 173, 217 (1980).

[4] K.K. Dwivedi, Radiat. Meas. 28, 145 (1997).
[5] M. Zamani, D. Sampsonidis, S. Charalambous, Nucl. Tracks 12, 125 (1986).

[6] T. Portwood, D.L. Henshaw, Nucl. Tracks 12, 105 (1986)

[7] D. Sinha, S. Ghosh, A. Srivastava V.G. Dedgaonkar, K.K. Dwivedi, Radiat. Meas. 28, 145 (1997).

[8] S.A. Durrani, R.K. Bull, Solid State Nuclear Track Detections. Principles, Methods and Applications, Pergamon Press, Oxford (UK) 1987.

[9] B. Jaleh, P. Parvin, K. Mirabaszadeh, M. Katouzi, Radiat. Meas. 38, 173 (2004)

[10] P. Parvin, B. Jaleh, N.A. Sheikh, Radiat. Meas. 40, 775 (2005)

[11] N. Dwaikat, T. Iida, F. Sato, Y. Kato, I. Ishikawa, W. Kada, A. Kishi, M. Sakai, Y. Ihara, Nucl. Instrum. Methods Phys. Res. A 572, 826 (2007)

[12] J. Tauc, Amorphous and Liquid Semiconductors, Plenum Press, New York 1974, p. 159.

[13] M.Y. Nadeem, T.B. Sadhana, M. Altaf, M.A. Chaudhry, J. Res. (Sci.), 15, 245 (2004).

[14] M.-S. Oh, D.-K. Hwang, D.-J. Seong, H.-S. Hwang, S.-J. Park, E.D. Kim, J. Electrochem. Soc. 155, D599 (2008)

[15] M.D. Migahed, H.M. Zidan, Curr. Appl. Phys. 6, 91 (2006) 